\title{
A novel method to enhance the sensitivity for two-degrees-of-freedom straightness measurement
}

\author{
Cuifang Kuang ${ }^{1,3}$, En Hong ${ }^{2}$, Qibo Feng ${ }^{1}$, Bin Zhang ${ }^{1}$ and \\ Zhifeng Zhang ${ }^{1}$ \\ ${ }^{1}$ School of Science, Beijing Jiaotong University, Beijing 100044, People's Republic of China \\ ${ }^{2}$ Mechanical Engineering Department, University of Michigan, Ann Arbor, MI 48109-2125, \\ USA \\ ${ }^{3}$ School of Information Science and Technology, Beijing Institute of Technology, \\ Beijing 100081, People's Republic of China \\ E-mail: kcf0424@sina.com
}

Received 16 March 2007, in final form 12 September 2007

Published 1 November 2007

Online at stacks.iop.org/MST/18/3795

\begin{abstract}
A low cost and high precision system which can measure two-degrees-of-freedom straightness simultaneously is proposed based on a laser collimator. In the system, a miniature fiber coupled diode laser generates a collimating beam. The sensitivity of the straightness error measurement is magnified by a factor of 4 compared with the lateral movement of the retro-reflector itself after the multi-reflections. A Renishaw ML10 laser interference measurement system was used to verify the developed system in a series of experiments in the laboratory environment. Experimental results agree with the expected results. The two-dimensional straightness error measurement had linear correlativity close to 1 and the standard one-to-one deviation error was better than $0.32 \mu \mathrm{m}$ obtained over a travel range of $600 \mu \mathrm{m}$.
\end{abstract}

Keywords: straightness errors, two-degrees-of-freedom, laser measurement, laser collimated, linear guide way

(Some figures in this article are in colour only in the electronic version)

\section{Introduction}

Straightness measurement is an important part in modern machining tool calibration and also an essential criterion for the performance of machine tools. It is mostly composed of the straightness error measurement of the work surface and the linear stage or the self-correcting moving mechanism. Optical measurement of straightness errors has been widely used in the field of engineering metrology. There are mostly three methods that use the laser as a reference.

The first method is laser interferometry measurement based on wavelength [1-13], which includes four kinds in turn: (1) the method using the Wollaston prism [1-4], (2) the method using a triangular prism [5, 6], (3) the method using a holographic grating $[7,8]$ and (4) the method using moiré fringes [9-12]. The laser interferometry method has the advantages of precision and anti-interference by trade-off in system complexity and expense.

The second method is polarimetry optical modulation measurement using laser polarization status as the reference [14-16]. The straightness error can be obtained by analyzing the energy variation. Therefore, the laser device's power fluctuation affects the measurement accuracy evidently. A stable laser source is the key element in this method.

The third is the laser beam straight datum (collimated laser) measuring method as used in these papers [17-32]. Most current research focuses on this method which includes the reflection confocal optical method [17-19], energy center detection [20-25], energy change detection [26, 27], autocollimator angular calculation [28] and retro-reflector method [29-32]. 


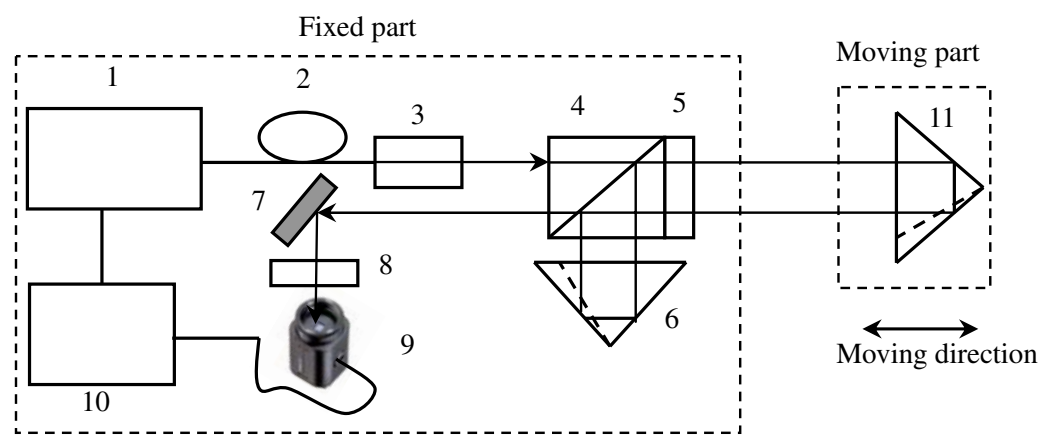

1-laser diode; 2-single-mode fiber; 3-collimating lenses; 4-polarization beam splitter; 5-quarter wave plate; 6,11-retroreflector, 7-plane reflector, 8- narrow band pass filter; 9-CCD camera; 10- processor module

Figure 1. Schematic diagram of the measuring system of two-dimensional straightness errors.

In this paper, a diode laser coupled with a singlemode optical fiber was applied as a collimating light. It has the advantage of being low cost, fairly stable, small capacity, low power consumption. The straightness can be derived by detecting the center of the laser beam $[21,24,25]$ on a CCD camera after it reflects back from retro-reflectors [30-32]. Specially designed optical pass could change the polarization of the laser beam four times and magnify the lateral movement of the retro-reflector twice. This novel method improves the sensitivity of the real-time two-dimensional straightness error measurement. The goal of this research is to develop an innovative laser straightness measurement system based on the concepts introduced above and laser alignment technology.

\section{Measurement principle}

The following section will describe the principle of measurement used in the system and the system configuration will be listed as well. Figure 1 shows the overall schematic of the system. The system consists of a fixed unit and a moving unit. The fixed unit is placed on the axis of the measurement. The moving unit should be rigidly mounted onto the moving object or target. A collimated laser source (1) and CCD camera are mounted on the fixed unit. The laser beam emitted from the end of the single-mode fiber (2) is collimated with a parallel beam through the collimation lenses (3). The collimated laser beam passes through the polarizing beam splitter (PBS) (4) to change into a vertical linear polarization beam, following which it passes through a quarter wave plate (5). The orientation of the fast axis of the quarter wave plate is set to $45^{\circ}$ with reference to the vertical plane. During the first pass, the quarter wave plate changes the linear polarization state to circular. Then the beam hits the target retro-reflector (11) on the moving part and returns to the PBS (4) after passing through the wave plate (5) again. The polarization state of the measurement beam has been shifted by $90^{\circ}$ with respect to its original state in order to be in a horizontal linear polarization status. Therefore, the beam could only be reflected on the hypotenuse of the PBS (4) instead of passing through the PBS. Then the beam reflects off the second retroreflector (6) and makes its second pass toward the moving

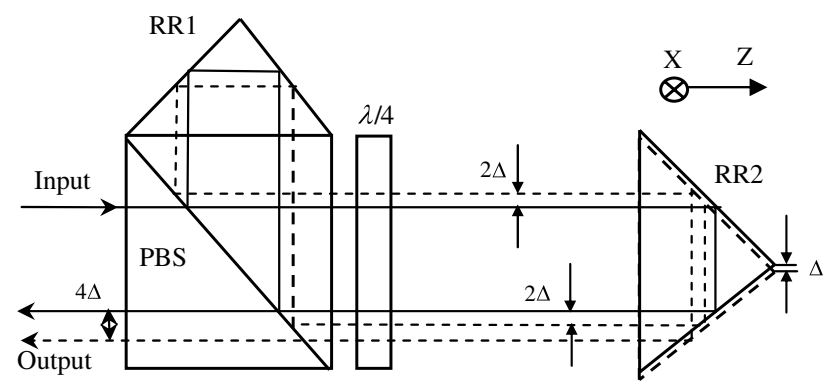

Figure 2. Relation between the straightness error and the reflecting beam.

retro-reflector (11). The same change in the polarization state happens during the second pass, transforming the beam to its original vertical linear polarization state. After that the beam is reflected by the plane mirror (7), and passes through the narrow optical band pass filter (8) to the CCD detector (9). The retro-reflector's lateral movements are perpendicular to the direction of movement, which can be detected by the $\mathrm{CCD}$, reflecting the straightness errors of the linear guide way. Power for both the laser and the CCD driver comes from the processor module (10). The signals received by the $\mathrm{CCD}$ are sent to the data processing unit. After the post processing, the two-dimensional straightness errors can be obtained. The actual system configuration is listed below. The laser unit (1) is a fiber coupled laser diode module NT55-938 (635 nm, $1 \mathrm{~mW}$ ) made by Edmund Optics. The collimating lens is a Data Optics objective lens MO-0010 10X. The quarter wave plate (5) is an Edmund Optics NT43-698. The PBS (4) is an Edmund Optics NT45-200. The retroreflectors (11) and (6) are Edmund Optics NT 43-297. The CCD camera is a WAT $902 \mathrm{H} 31 / 3$ inch monochrome camera. The frame grabber (10) is a National Instruments 1405 board.

\section{System measurement sensitivity analysis}

The relationship between the straightness error and the beam's displacement on the CCD is derived below. Figure 2 shows that if the second retro-reflector RR2 mounted on the moving unit has a moving error $\Delta$ in the vertical direction, the reflected 


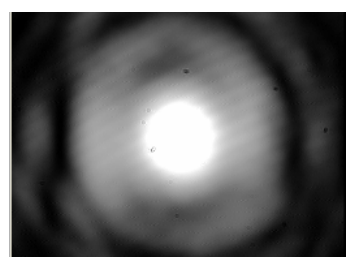

(a)

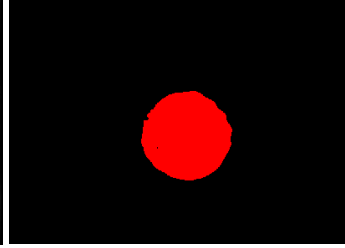

(b)

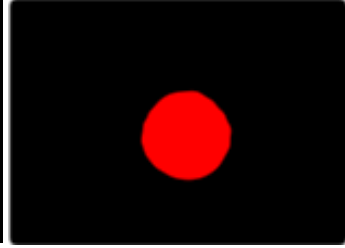

(c)

Figure 3. Beam on the CCD: $(a)$ original beam spot, $(b)$ above threshold without a low-pass filter level, $(c)$ above threshold with a low-pass filter level.
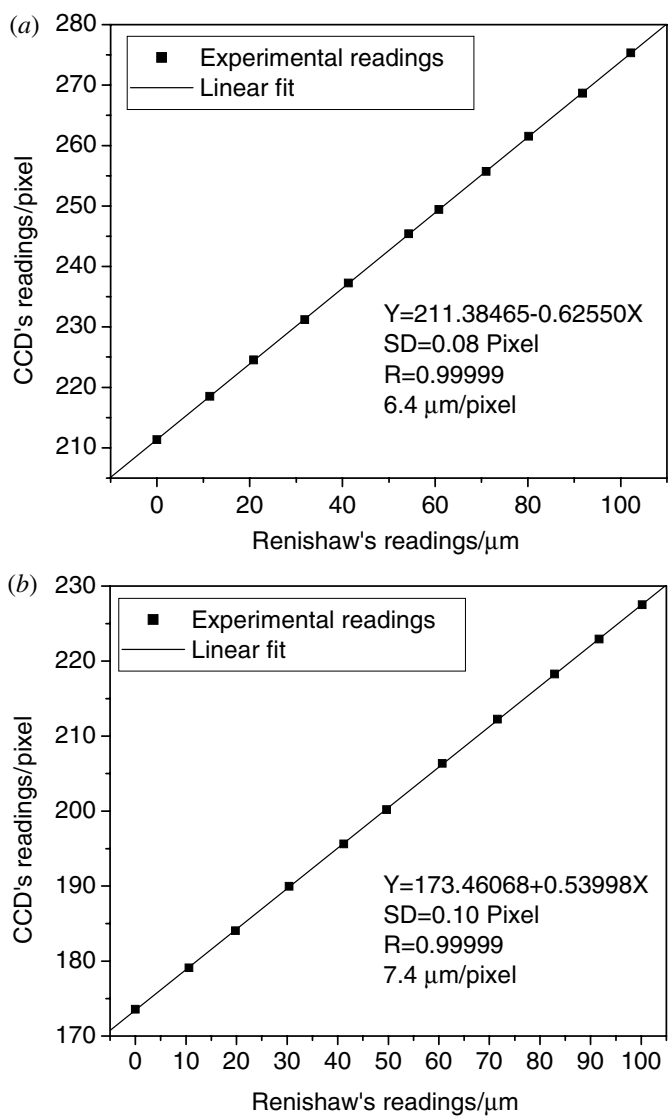

Figure 4. (a) Sensitivity verification experiment for the horizontal direction and $(b)$ sensitivity verification experiment for the vertical direction.

beam will have a movement of $2 \Delta$ after passing through the RR2, increasing to $4 \Delta$ after the second pass. Finally, the displacement of the beam center detected by the CCD in the vertical direction is four times that of the original movement $\Delta$ of the RR2. The same relationship can be derived in the horizontal direction easily. The formula is shown below:

$$
X=4 \Delta_{x}, \quad Y=4 \Delta_{y},
$$

where $\Delta_{x}$ and $\Delta_{y}$ are the movements of the moving unit RR2 in the vertical and horizontal directions and $X$ and $Y$ signify the beam center position changes on the CCD. Equation (1) shows that the system measurement sensitivity improves twice over that of the reference works [15, 18, 19]. It is also very important to diminish the noise of the signal using data processing techniques to find the accurate center of the beam
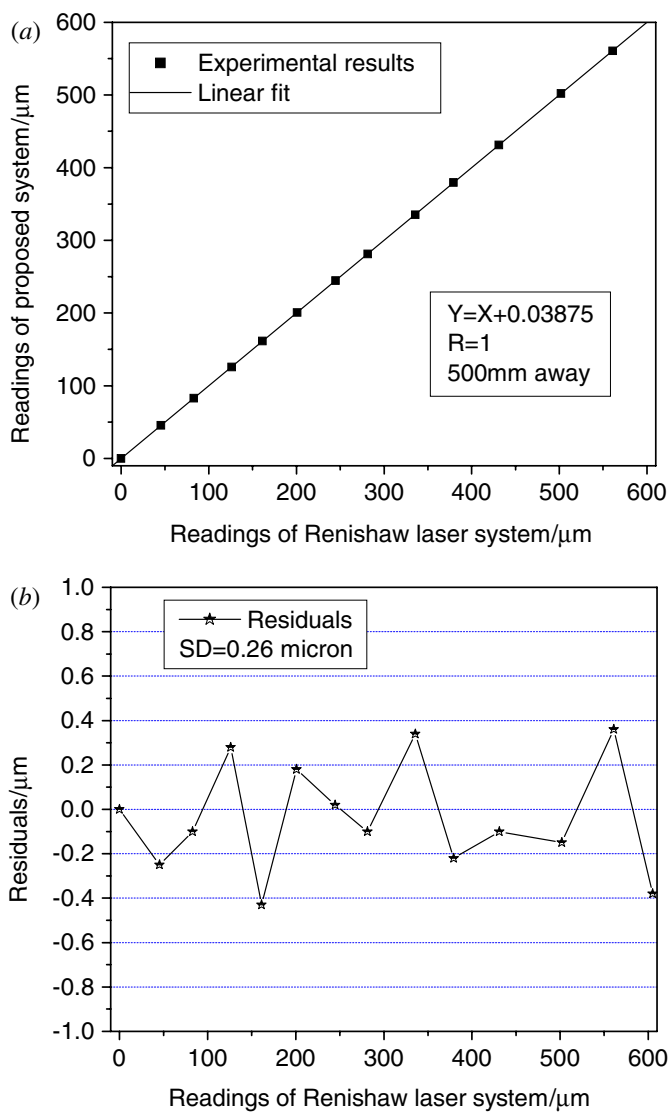

Figure 5. (a) Calibration results for the horizontal direction and (b) residuals for the horizontal direction calibration.

spot on the detector including its deviation and displacement. The absolute positions of the $X$ and $Y$ axes of the beam spot are given by

$$
X=\left(\sum_{i=1}^{N_{x}} X_{i} G_{i}\right) / \sum_{i=1}^{N_{x}} G_{i}, \quad Y=\left(\sum_{j=1}^{N_{y}} Y_{j} G_{j}\right) / \sum_{j=1}^{N_{y}} G_{j},
$$

where $N_{x}$ and $N_{y}$ are the numbers of elements in the $X$ and $Y$ CCD arrays, $X_{i}$ and $Y_{j}$ are the $X$ and $Y$ axes coordinates, and $G_{i}$ and $G_{j}$ are the gray levels of every element on the CCD array's $X$ and $Y$ directions. Suppose the CCD position resolution can reach sub-pixel $0.1 \mu \mathrm{m}$ by the image processing techniques; then the system's theoretical measurement resolution is $0.025 \mu \mathrm{m}$ based on equation (1). 

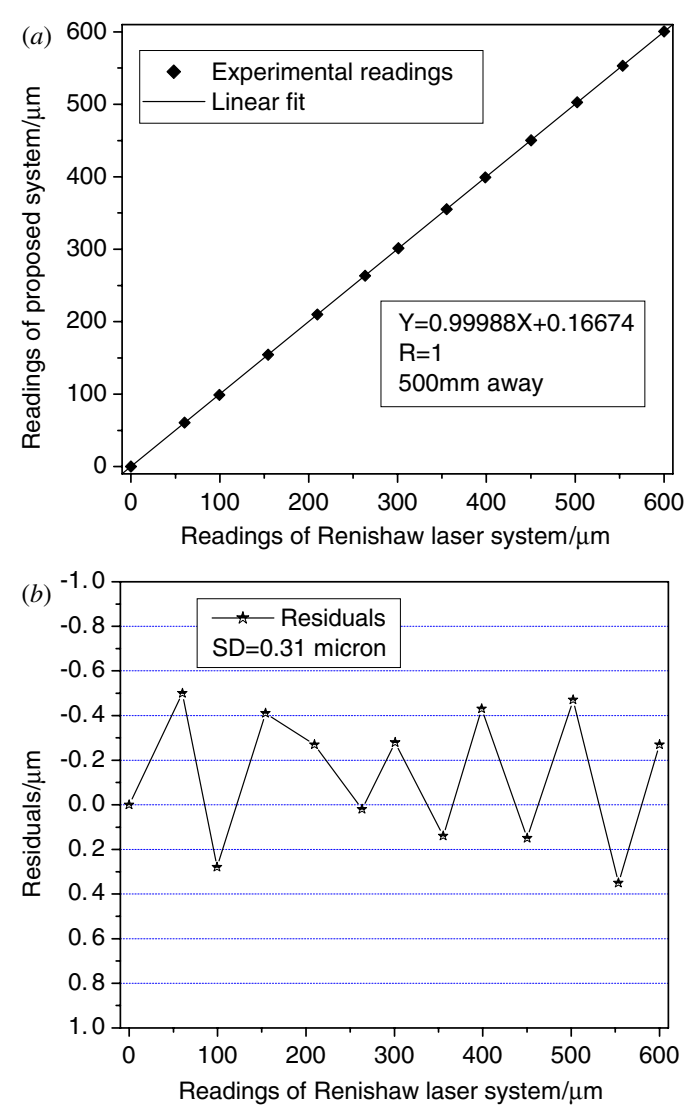

Figure 6. (a) Calibration results for the vertical direction and (b) residuals for the vertical direction calibration.

\section{Experiments and analysis}

A series of experiments were done under laboratory conditions (temperature: $(23 \pm 1){ }^{\circ} \mathrm{C}$, air pressure: $(765 \pm 2) \mathrm{mmHg}$, humidity: $<63 \%$ ) to verify the system's feasibility, stability and accuracy. Figure 3(a) shows the original light spot's shape on the CCD from the reflected beam of the target retro-reflector. The blurs were caused by the optical system diffraction, random dithering, the electronic noise of the device, etc. Figure 3(b) shows the light spot's shape after setting a gray threshold. In order to smooth the edge of the beam spot, we adopted a low-pass filter. The low-pass filter is a filter that passes low frequency signals, but attenuates (reduces the amplitude of) signals with frequencies higher than the cutoff frequency. Figure 3(c) shows the spot's shape after introducing the low-pass filter and setting a gray threshold. The noise has been suppressed greatly and the edge of the beam spot is smoother than formerly. The Renishaw ML10 laser interferometer was used here to calibrate and compare the measurement results. The linear measurement resolution of the Renishaw ML10 is $0.001 \mu \mathrm{m}$ and the measurement accuracy is $\pm 0.7 \mathrm{ppm}$ (with EC10 compensation). The ML10 system accuracy is quoted to the internationally recognized 95\% confidence level $(k=2)$.

\subsection{Calibration experiments}

The RR2 was mounted on a manual precision optical motion stage. Figure 4 shows that when the target retro-reflector
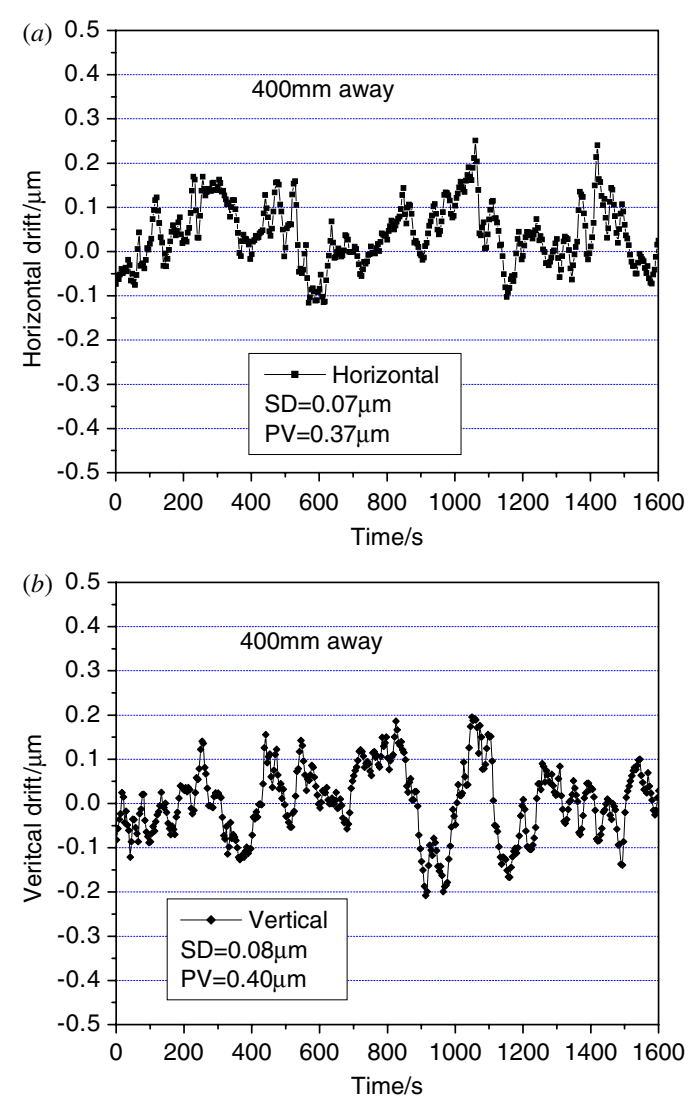

Figure 7. (a) Stability for the horizontal direction and (b) stability for the vertical direction.

RR2 moves from 0 to $100 \mu \mathrm{m}$ in the vertical and horizontal directions by reading Renishaw's interferometer system's results, the center of the laser beam spot moved 62.505 pixels and 53.998 pixels in the horizontal and the vertical directions respectively. The pixel size of the CCD is $6.4 \times 7.4 \mu \mathrm{m}^{2}$, so the displacements are $400.03 \mu \mathrm{m}$ in the $X$ direction and $399.59 \mu \mathrm{m}$ in the $Y$ direction. The experimental results verified that the sensitivity of the measurement is up to four times the actual movement shown on the Renishaw system, which agrees with the result of the theoretical calculation.

Figure 5(a) shows the calibration result in the range of $0-60 \mu \mathrm{m}$ in the horizontal direction. Its linear correlation is almost 1 . The error of the corresponding points is between $-0.43 \mu \mathrm{m}$ and $0.36 \mu \mathrm{m}$ and the standard deviation $\sigma_{h}$ is $0.26 \mu \mathrm{m}$, as shown in figure $5(b)$. The calibration result in the vertical direction is demonstrated in figure $6(a)$. The linear correlation is also close to 1 . The error of the corresponding points is between $-0.5 \mu \mathrm{m}$ and $0.35 \mu \mathrm{m}$ and the standard deviation error $\sigma_{h}$ is $0.31 \mu \mathrm{m}$, as shown in figure $6(b)$. The experimental results show that the two-dimensional residual errors are less than $0.32 \mu \mathrm{m}$.

\subsection{Stability experiments}

A system stability test was performed to validate the measurement stability and accuracy. The target RR2 is $400 \mathrm{~mm}$ away; the results of stability experiments are shown in figure 7. The laser beam drifts in the horizontal and vertical directions are about $\pm 0.2 \mu \mathrm{m}$, and the standard deviation 

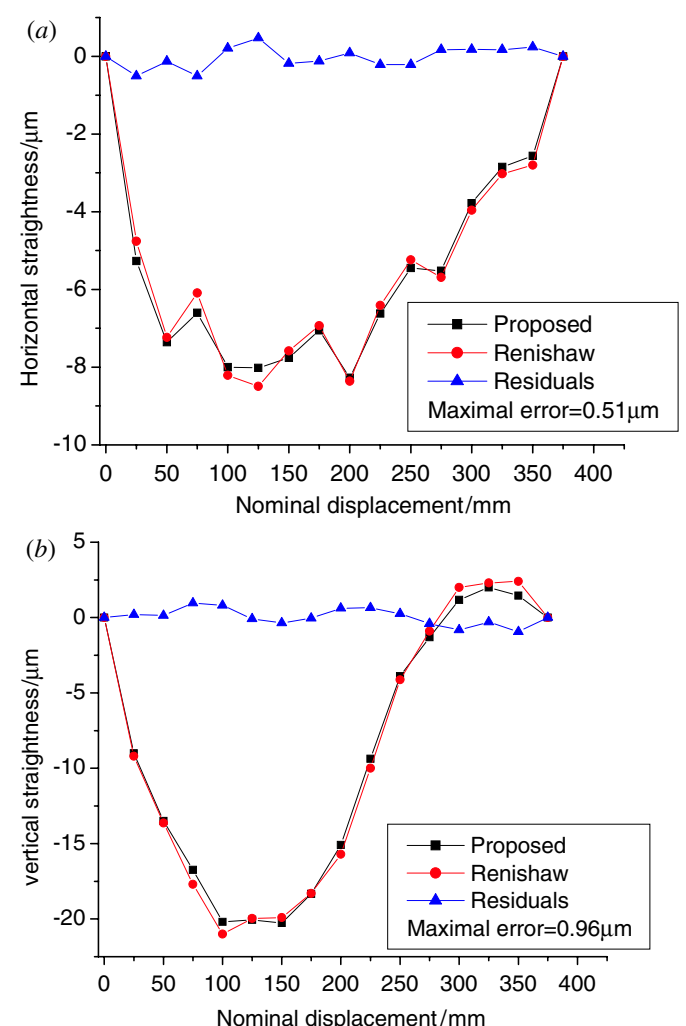

Figure 8. (a) Comparison results of the horizontal straightness between the proposed system and the Renishaw system and $(b)$ comparison results of the vertical straightness between the proposed system and the Renishaw system.

is about $0.08 \mu \mathrm{m}$ during the $25 \mathrm{~min}$ of measurement. The small drifts guaranteed the high stability of the system under laboratory measurement conditions.

\subsection{Comparison experiments}

A comparison test of straightness errors was performed on a precision linear stage. The characteristic curves of straightness comparison are shown in figures $8(a)$ and $(b)$ in which the residuals of the point-to-point measurements of the horizontal and vertical straightness measurements are also plotted. The experimental results were processed by a two-point method. The results show that the maximum difference of the proposed method and Renishaw measurement system is $0.51 \mu \mathrm{m}$ in the horizontal direction and $0.96 \mu \mathrm{m}$ in the vertical direction. This proves good consistency between the two measuring systems. From the calibration experimental results, it can be seen that the standard deviation $(\sigma)$ of the proposed system is less than $0.32 \mu \mathrm{m}$. So, the measurement uncertainty $(3 \sigma)$ of the system is less than $1 \mu \mathrm{m}$. The system uncertainty is verified in comparison experiments.

\section{Error source analysis}

The proposed system error sources are as follows. (1) The system is sensitive to changes in the laser beam pointing direction, and this sensitivity increases with range. For example, if the pointing direction of the output beam shifts by 0.1 arcsec, then the shift in straightness reading from the electronic target will be $0.5 \mu \mathrm{m}$ at $1 \mathrm{~m}$ range. (2) Internal optical reflections and dust and dirt on glass components can disrupt the position of the beam 'center of intensity' and light in the halo may miss the target altogether. This makes it important to keep the optical surfaces clean in order to maintain optimum measurement accuracy. (3) The measurement accuracy also depends on both the intensity of the stray beam and where it strikes the target. So, steps need to be taken to eliminate stray reflections, or to ensure that they remain stable during measurement. (4) This system also suffers from air turbulence noise, and the problem is caused by beam bending; the errors increase at longer range.

\section{Conclusions}

A novel system for simultaneously measuring two-degrees-offreedom straightness errors is developed based on a collimated laser beam. The system configuration is uncomplicated and provides good performance. The relationship between the straightness error and the beam displacement was derived. The total straightness error was amplified by a factor of 4. Post-image processing techniques such as low-pass filter and proper gray thresholds improved the sensitivity of the measurement greatly. The experimental results agree with the expected theoretical results. The system's standard deviation of the two-dimensional straightness errors is less than $0.33 \mu \mathrm{m}$. This system can be used in two-dimensional straightness error measurement in both off-line and real-time scenarios, and monitor the two-dimensional small translation in a workplace or target space observation. It can also be used as an optional feedback monitor in some real-time closed-loop control systems in many applications such as $x-y$ stages, precision machine tools, coordinate measuring machines, etc. According to the principle of this paper, the measurement sensitivity can be further improved by increasing the multiple reflections. However, these changes will mean larger energy loss, extra air turbulence interference and more optical components.

\section{Acknowledgments}

The research was supported by the doctoral innovation foundation of Beijing Jiaotong University with no. 48003 and the National Natural Science Foundation of China under grant no. 50675017.

\section{References}

[1] Baldwin R R 1974 Interferometer system for measuring straightness and roll US Patent 3790284

[2] Lin S-T 2001 A laser interferometer for measuring straightness Opt. Laser Technol. 33 195-9

[3] Chianey R J 1994 Two component straightness interferometer apparatus for measurement movements of parts of a machine US Patent 5337145

[4] Chen Q, Lin D, Wu J, Yan J and Yin C 2005

Straightness/coaxiality measurement system with transverse Zeeman dual-frequency laser Meas. Sci. Technol 16 2030-7 
[5] Zhang J and Cai L 1998 Interferometric straightness measurement system using triangular prisms Opt. Eng. 37 1785-9

[6] Wu C-M 2004 Heterodyne interferometric system with subnanometer accuracy for measurement of straightness Appl. Opt. 43 3812-6

[7] Yamauchi M and Matsuda K 1994 Interferometric straightness measurement system using a holographic grating Opt. Eng. 33 1078-83

[8] Celaya M, Rizo I and Mercado E 1989 Hologram interferometer to calibrate and measure the straightness in micropositioning equipment Proc. SPIE-Int. Soc. Opt. Eng. 1036 44-51

[9] Burch J M and Williams D C 1977 Varifocal moiré zone plates for straightness measurement Appl. Opt. 16 2445-50

[10] Zhang X 2004 Measurement method of spatial straightness error using non-diffracting beam and moiré-fringe technology J. Opt. A: Pure Appl. Opt. 6 121-6

[11] Sakuma H H and Wada H 1987 Straightness measurement using the heterodyne moiré method Precis. Eng. 9 19-22

[12] Bin Z 2004 Digital moiré fringe-scanning method for centering a circular fringe image Appl. Opt. $432833-9$

[13] Ma L, Wang L and Cao T 2007 A large-scale laser plane calibration system Meas. Sci. Technol. 18 1768-72

[14] King R J and Raine K W 1981 Polarimetry applied to alignment and angle measurement Opt. Eng. 20 39-43

[15] Yin C, Chen W, Fang Z and Liang J 1991 Two-dimensional automatic straightness measurement system based on optical activity Opt. Eng. 30 480-2

[16] Rzepka J 2005 Zeeman laser for straightness measurements. Optical measurement systems for industrial inspection IV Proc. SPIE 5856 ed W Osten, C Gorecki and E Novak (Bellingham, WA: SPIE) pp 625-31

[17] Matsuda K, Eiju T and Roy M 1999 Straightness measurements using a reflection confocal optical system Proc. SPIE 3749 589-90

[18] Matsuda K, Roy M and Eiju T 1999 Straightness measurements by use a reflection confocal optical system Appl. Opt. 38 5310-8
[19] Matsuda K 2002 Straightness measurements with a reflection confocal optical system-an experimental study Appl. Opt. 41 3966-70

[20] Shimizu S, Lee H and Imai N 1994 Simultaneous measuring of table motion errors in 6 degrees of freedom Int. J. Japan Soc. Precis. Eng $28273-4$

[21] Fan K C 2000 Laser straightness measurement system using optical fiber and modulation techniques Int. J. Mach. Tools Manuf. 40 2073-81

[22] Zhang X 2002 Study of the tolerance of the non-diffracting beam to laser beam deflection J. Opt. A: Pure Appl. Opt. $478-83$

[23] Whitford B G 1972 Two-aperture method of producing light beam with odd-symmetry for alignment and straightness measurements Appl. Opt. 11 2069-74

[24] Hao Q and Li D 2002 High-accuracy long distance alignment using single-mode optical fiber and phase plate Opt. Laser Technol. 34 287-92

[25] Hao Q 1998 Study on large scale form and position errors measurement Proc. SPIE Int. Soc. Opt. Eng. 3558 214-8

[26] Fan K-C, Chu C-L and Liao J-L 2003 Development of a high-precision straightness measuring system with DVD pick-up head Meas. Sci. Technol. 14 47-54

[27] Schwarz W 1996 Straightness measurements for accelerator structures Proc. 4th Int. Workshop on Accelerator Alignment (IWAA95) (KEK-Proc.95-12) pp 477-90

[28] Virdee M S 1995 Non-contacting straightness measurement to nanometer accuracy Int. J. Mach. Tools Manuf 35 157-64

[29] Huang P S and Ni J 1995 On-line error compensation of coordinate measurement machines Int. J. Mach. Tools Manuf 35 725-38

[30] Feng Q, Zhang B and Kuang C 2004 A straightness measurement system using a single-mode fiber-coupled laser module Opt. Laser Technol 36 279-83

[31] Kuang C, Feng Q and Zhang B 2005 A four-degree-of-freedom laser measurement system (FDMS) using a single-mode fiber-coupled laser module Sensors Actuators A 125 100-8

[32] He J 1998 Development of a six-degree-of-freedom laser measurement system for machine geometric error measurement PhD Dissertation Ann Arbor, University of Michigan 